\title{
Influence of trehalose 6,6'-dimycolate (TDM) during mycobacterial infection of bone marrow macrophages
}

\author{
Jessica Indrigo, Robert L. Hunter, Jr and Jeffrey K. Actor
}

Department of Pathology, Program in Molecular Pathology, University of Texas-Houston Medical School, MSB 2.214, 6431 Fannin, Houston, TX 77030 USA
Author for correspondence: Jeffrey K. Actor. Tel: +1 713500 5344. Fax: +1 7135000730. e-mail: Jeffrey.K.Actor@uth.tmc.edu

The relative role of surface lipids in the innate macrophage response to infection with mycobacteria remains unknown. Trehalose 6,6'-dimycolate (TDM), a major component of the mycobacterial cell wall, can elicit hypersensitive as well as T-cell-independent foreign body responses. The T-cellindependent contribution of TDM to the primary macrophage response to mycobacterial infection was investigated. Bone-marrow-derived macrophages isolated from C57BL/6 mice were infected with native Mycobacterium tuberculosis (MTB) or with MTB delipidated using petroleum ether extraction methods. The removal of surface lipids caused decreased bacterial survival in macrophages, but there was no loss of bacterial growth in broth culture. Bacterial survival within macrophages was restored upon reconstitution of the bacteria with purified TDM. The cytokine and chemokine parameters of the macrophage responses were also investigated. The amounts of IL-1 $\beta$, TNF- $\alpha$, IL-6 and MIP-1 $\alpha$ produced were significantly reduced following delipidation, but were restored upon reconstitution with TDM. The amount of IL-12 produced, but not the amount of IL-10 produced, was also significantly reduced upon macrophage infection with delipidated MTB. Furthermore, nitric oxide responses were not impaired upon infection with delipidated MTB, suggesting that intracellular survival and macrophage secretion of cytokines and chemokines are differentially controlled. These studies indicate that TDM is a major component contributing to the innate macrophage responses to MTB infection.

Keywords: Mycobacterium tuberculosis, cytokines, chemokines, cord factor

\section{INTRODUCTION}

Characterization of the cell wall of Mycobacterium tuberculosis (MTB), the causative agent of tuberculosis, is the focus of intense research. The lipid and lipidcontaining components of this bacterium are present in unusual abundance and constitute $60 \%$ of the cell wall (Kolattukudy et al., 1997). The hydrophobicity bestowed upon MTB by its cell-wall lipids contributes to the tendency of the bacteria to aggregate into long serpentine cords when grown in broth culture (Bloch, 1950; Middlebrook et al., 1947). The lipid-rich cell wall confers an extremely low permeability barrier on MTB,

Abbreviations: BCG, Bacille Calmette-Guérin; BMM, bone-marrowderived macrophage; FBS, fetal bovine serum; MTB, Mycobacterium tuberculosis; TDM, trehalose 6,6'-dimycolate. contributing to its resistance to most common therapeutic agents (Jarlier \& Nikaido, 1994) as well as to its ability to survive within the hostile environment of the macrophage (Spargo et al., 1991).

Virulent MTB strains subjected to surface lipid extraction by petroleum ether treatment became avirulent and unable to form cords, but they retained their viability (Bloch, 1950). The petroleum ether fraction was termed the 'cord factor' and was later identified to be composed primarily of trehalose 6,6'-dimycolate (TDM; Noll et al., 1956). Silva et al. (1985) showed that TDM from the vaccine strain $M$. bovis Bacille Calmette-Guérin (BCG) modulated infection in Swiss mice. Mice infected with delipidated BCG, which lost TDM due to petroleum ether extraction, exhibited fewer granulomatous lesions and less intense delayed-type hypersensitivity than mice infected with native BCG 
(Silva et al., 1985). Furthermore, the viability of delipidated BCG was significantly reduced in all organs by 4 days after infection, whereas native BCG remained viable for at least 24 days. When delipidated BCG was reconstituted with purified TDM, the native phenotype was almost entirely restored (Silva et al., 1985).

Recent studies have demonstrated that TDM is able to reproduce many of the pathophysiological characteristics of MTB infection when injected intravenously into mice and rabbits, including the induction of hypersensitive-type pulmonary granulomas and the concurrent production of inflammatory cytokines, as well as increased procoagulant activity (Hamasaki et al., 2000; Perez et al., 1994, 2000; Yamagami et al., 2001). The inflammatory responses parallel, in part, responses identified during acute experimental infection with MTB, most notably those involved in and appearing during the formation of pulmonary granulomas (TNF$\alpha$, IL-1 $\beta$ and IL-6) (Actor et al., 2000; Watson et al., 2000). It has been suggested that the responses elicited by TDM during infection are regulated through a combination of T-cell-dependent and T-cell-independent mechanisms (Yamagami et al., 2001).

The contribution of MTB surface lipids, primarily TDM, to the survival of MTB and to the induction of Tcell-independent inflammatory responses warranted further investigation. We hypothesized that the removal of MTB surface lipids and of TDM would lead to alterations in the innate macrophage response, especially in the production of inflammatory mediators such as chemokines and nitric oxide. Non-covalently bound cell-wall lipids were removed from MTB by petroleum ether extraction methods; these delipidated organisms were used to infect purified populations of C57BL/6 bone-marrow-derived macrophages (BMMs). The survival of organisms was monitored, and the cytokine and chemokine responses were evaluated and compared to native organisms as well as to delipidated organisms reconstituted with purified TDM.

\section{METHODS}

BMMs. Six-week-old female C57BL/6 mice were obtained from The Jackson Laboratory (Bar Harbour, ME). BMMs were established as described by Falk (1994) and Sutterwala et al. (1997), with minor modifications. Femurs were flushed with PBS $\left(0 \cdot 2 \mathrm{~g} \mathrm{KCl} \mathrm{l}^{-1}, \quad 0 \cdot 2 \mathrm{~g} \mathrm{KH}_{2} \mathrm{PO}_{4} \mathrm{l}^{-1}, \quad 8 \cdot 0 \mathrm{~g} \mathrm{NaCl} \mathrm{l}^{-1}\right.$, $\left.1 \cdot 15 \mathrm{~g} \mathrm{Na}_{2} \mathrm{HPO}_{4} \mathrm{l}^{-1}, \mathrm{pH} 7 \cdot 4\right)$ and $3 \times 10^{7}$ cells were added to $75 \mathrm{~cm}^{2}$ tissue-culture flasks (Costar). Cells were grown in Eagle's minimal essential medium (EMEM; Sigma) containing $10 \%$ fetal bovine serum (FBS), $2 \mathrm{mM}$ glutamine, $10 \mathrm{ng}$ recombinant murine granulocyte/macrophage colony stimulating factor (GM/CSF) $\mathrm{ml}^{-1}$ (Chemicon), $100 \mathrm{U}$ penicillin $\mathrm{ml}^{-1}$ and $100 \mu \mathrm{g}$ streptomycin $\mathrm{ml}^{-1}$. Cells were incubated at $37{ }^{\circ} \mathrm{C}$ in $5 \% \mathrm{CO}_{2}$ overnight. Non-adherent cells were collected, incubated for 7 days and fed twice with additional medium containing GM/CSF. Finally, adherent cells were removed, washed and resuspended in Dulbecco's modified essential medium (DMEM; Sigma) containing $2 \%$ FBS. Cells were then adjusted to $1 \times 10^{6}$ cells $\mathrm{ml}^{-1}$ and added to 24 -well tissue culture plates (Costar) in a total volume of $1 \mathrm{ml}$.

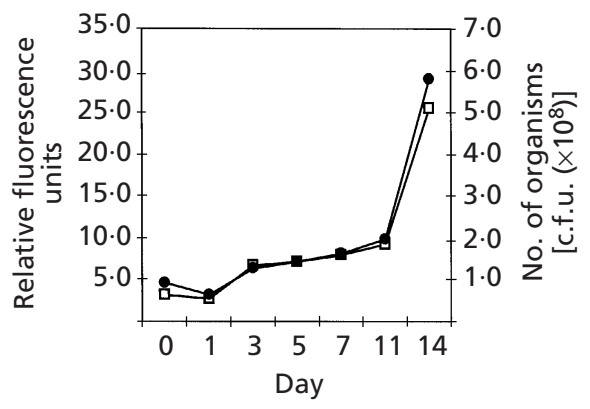

Fig. 1. Viability of MTB. Delipidation of MTB does not affect the viability and growth rate of organisms. No significant differences in growth in Dubos broth were seen through 14 days between the native organisms ( $)$ and organisms that were delipidated by petroleum ether treatment ( $\square$ ). Data represent incorporation of a fluorescent nucleic acid stain, expressed as relative fluorescence units ( \pm one SD) as related to conventional c.f.u. growth on Middlebrook 7H11 agar. Nearly $100 \%$ of both the native and delipidated organisms stained acid-fast (not shown).

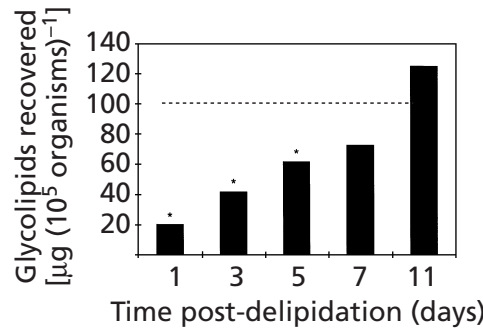

Fig. 2. Recovery of surface glycolipids following petroleum ether treatment. MTB was delipidated and grown in Dubos broth. Surface glycolipids were extracted with petroleum ether at the indicated times. Data are shown relative to native MTB that was grown under identical conditions. For the native organisms (indicated by the dotted line), 100.68 ( $\pm 11.13 \mathrm{SE}) \mu \mathrm{g}$ glycolipid were recovered $\left(10^{5} \text { organisms }\right)^{-1}$. Asterisks indicate statistically significant results, $P<0.05$.

Mycobacteria. MTB (Erdman, ATCC 35801) was cultured to the exponential phase of growth in Dubos broth (Difco) supplemented with $5 \%$ BSA and $7 \cdot 5 \%$ dextrose. For delipidated MTB, mycobacterial surface lipids were extracted with petroleum ether, as described by Silva et al. (1985). After the addition of petroleum ether (Sigma), the bacteria were vortexed vigorously for $2 \mathrm{~min}$, followed by $5 \mathrm{~min}$ incubation at room temperature. The culture was centrifuged at $500 \mathrm{~g}$ for $10 \mathrm{~min}$. The supernatant, which contained extracted material, was removed and the extraction process was repeated twice more. After the last extraction, the bacteria were washed to remove any residual petroleum ether; they were then resuspended in PBS. Petroleum ether extraction by these methods does not affect the viability nor the acid-fastness of organisms. Delipidated mycobacteria demonstrated no reduction in their viability (Fig. 1) through 14 days of growth in Dubos broth. Growth was measured by the incorporation of a fluorescent nucleic acid stain into the bacteria, using the BacLight kit (Molecular Probes) according to the manufacturer's directions. Relative fluorescence units $(485 \mathrm{~nm}$ excitation/527 nm 


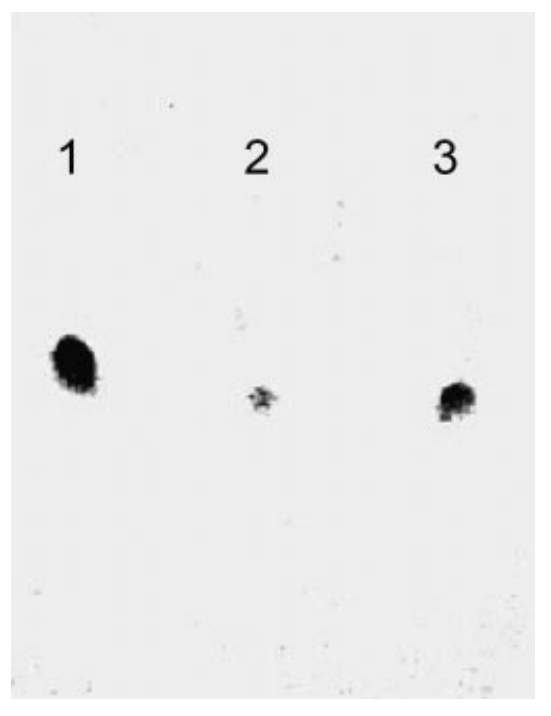

Fig. 3. TDM is the primary component of petroleum ether extracts of MTB. TLC was performed on silica gel 60A plates (Whatman). Lanes: $1,10 \mu \mathrm{l}(50 \mu \mathrm{g})$ TDM standard; $2,10 \mu \mathrm{l}$ extracted material; 3, $20 \mu \mathrm{l}$ extracted material. The plate was developed in chloroform/methanol (9:1) and visualized by spraying with $5 \%$ sulfuric acid containing $10 \% \alpha$-naphthol followed by charring for $10 \mathrm{~min}$.

emission) were measured on a Fluoroskan Ascent CF (Thermo Labsystems) fluorometer. Delipidated organisms retained their acid-fastness (not shown), as indicated by staining with the fluorescent Bacto TB Auramine M kit (Difco). Organisms treated with petroleum ether reconstituted TDM following delipidation (Fig. 2). Delipidated organisms were monitored for their recovery of surface glycolipids; the level of surface glycolipids recovered was statistically indistinguishable from native organisms by day 7 post-delipidation.

The extracted material was analysed by TLC; TDM was the primary component extracted (Fig. 3). This is in concert with the HPLC analysis performed by Silva et al. (1985), who found that a similar petroleum ether extract of $M$. bovis BCG contained $95 \%$ TDM, with small quantities of free mycolic acid glycerides and hydrocarbons also present. Reconstitution of delipidated MTB occurred by the addition of a $0.01 \%$ $\left(50 \mu \mathrm{g} \mathrm{ml}^{-1}\right)$ solution of purified TDM (Sigma; $100 \%$ pure by TLC, $>98 \%$ as $6,6^{\prime}$-mycolate esters) in petroleum ether (Silva et al., 1985). The solvent was evaporated and the bacteria were resuspended in PBS.

Prior to infection of the BMM monolayers, the bacteria were adjusted to $5 \times 10^{6}$ bacteria $\mathrm{ml}^{-1}$ in DMEM containing $2 \%$ FBS and sonicated to disperse clumps. Serial dilutions were plated onto Middlebrook 7H11 agar (Remel) to confirm the infectious dose. The c.f.u. values were enumerated after incubation of the plates at $37^{\circ} \mathrm{C}$ for 21 days.

Infection of BMMs. Matured BMMs were infected $24 \mathrm{~h}$ after seeding (m.o.i. 5:1). Immediately before their use, the monolayers were washed extensively with PBS. One millilitre of native $\mathrm{MTB}$, delipidated $\mathrm{MTB}$, delipidated MTB reconstituted with purified TDM or medium alone was added to the macrophages and the infection was allowed to proceed for $4 \mathrm{~h}$ at $37^{\circ} \mathrm{C}$, with gentle rotation. Cells were infected in triplicate wells. Upon completion of the infection period, cells were washed to remove extracellular bacteria. Fresh DMEM containing $2 \%$ FBS was then added to the wells.
The BMMs were lysed with 0.05\% SDS at 4, 24 and $96 \mathrm{~h}$ after infection to assess bacterial survival. Serial dilutions of the lysates were plated onto Middlebrook 7H11 agar and incubated at $37^{\circ} \mathrm{C}$ for 21 days for enumeration of the c.f.u. values.

Measurement of inflammatory mediators. After infection with mycobacteria, the production of IL- $1 \beta$, IL-6, IL-10, IL-12, TNF $-\alpha$ and MIP $-1 \alpha$ by the BMMs was measured in culture supernatants by ELISA. Measurements were performed with specific ELISA DuoSet kits (R\&D Systems), following the manufacturer's directions. Costar 96-well high-binding plates were coated with capture antibody overnight. Plates were washed three times with wash buffer $(0.05 \%$ Tween 20 in PBS). Blocking buffer (1\% BSA, $5 \%$ sucrose and $0.05 \% \mathrm{NaN}_{3}$ in PBS) was added to the wells for $2 \mathrm{~h}$. After three washes, $100 \mu \mathrm{l}$ of the cell-culture supernatant was added and the plates were incubated for a further $2 \mathrm{~h}$. After this time, the plates were washed extensively and biotin-conjugated secondary antibodies were added to the wells. Finally, the plates were incubated for $2 \mathrm{~h}$, washed and then developed using streptavidin-horseradish peroxidase and the TMB Microwell Peroxidase Substrate (Kirkegaard and Perry Laboratories). Absorbance was read at $450 \mathrm{~nm}$, with a background correction set at $570 \mathrm{~nm}$, on an ELISA plate reader (Molecular Devices). The mean amount of each inflammatory mediator present (from triplicate wells) was calculated based on a standard curve constructed for each assay using recombinant murine controls ( $\mathrm{R} \& \mathrm{D}$ Systems). The limit of sensitivity was 5-10 $\mathrm{pg} \mathrm{ml}^{-1}$ for each assay.

Nitric oxide production was measured using the colorimetric Griess assay, which detects nitrite in culture supernatants. Griess reagent $(100 \mu \mathrm{l} ; 0 \cdot 1 \% \mathrm{~N}$-1-napthylenediamine $\mathrm{HCl}$ in water and $1 \%$ sulfanilamide in $2.5 \%$ phosphoric acid) was added to $100 \mu \mathrm{l}$ of the BMM culture supernatants collected at various time points after infection. The plates were incubated at room temperature for $15 \mathrm{~min}$ and were protected from light. Absorbance values were read at $550 \mathrm{~nm}$ on a Molecular Devices plate reader. The absorbance values obtained for the culture supernatants from uninfected cells were subtracted from the absorbance values obtained for the experimental groups.

Statistical analysis. All experiments were performed in triplicate wells using BMMs obtained from three mice; growth curves and the measurement of molecular mediators are representative of three independent experiments. The differences between groups were analysed for significance using Student's $t$-test. A $P$ value $\leqslant 0 \cdot 05$ was considered significant.

\section{RESULTS}

\section{Decreased survival of delipidated MTB in C57BL/6 BMMs}

BMMs from C57BL/6 mice were infected with native MTB, delipidated MTB or delipidated MTB reconstituted with TDM. The survival of the bacteria and the production of inflammatory mediators was measured over time. The survival of intracellular mycobacteria was assessed by enumeration of the c.f.u. value at 4,24 and $96 \mathrm{~h}$ after infection (Fig. 4). At $96 \mathrm{~h}$ after infection, the viability of the delipidated organisms in the BMMs had decreased significantly $(P<0.001)$ when compared to native MTB; reconstitution of the delipidated organisms with purified TDM completely restored their viability. There was no significant difference in the viability of the native and reconstituted groups. 


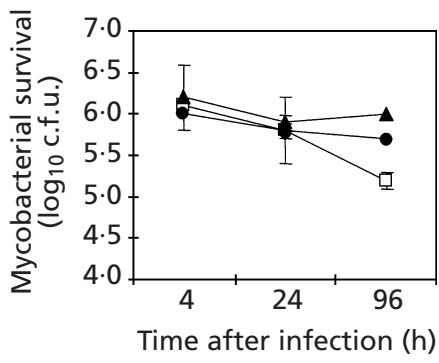

Fig. 4. Survival of mycobacteria in BMMs. C57BL/6 BMMs were infected with native MTB (O), delipidated MTB ( $\square$ ) or delipidated MTB reconstituted with $\operatorname{TDM}(\boldsymbol{\Delta})$ at an m.o.i. of $5: 1$. Mycobacterial survival was assessed by enumerating the c.f.u. values from plates seeded at 4, 24 and $96 \mathrm{~h}$ after infection. Data are expressed as the mean \pm one SD; three wells were assayed for each time point.

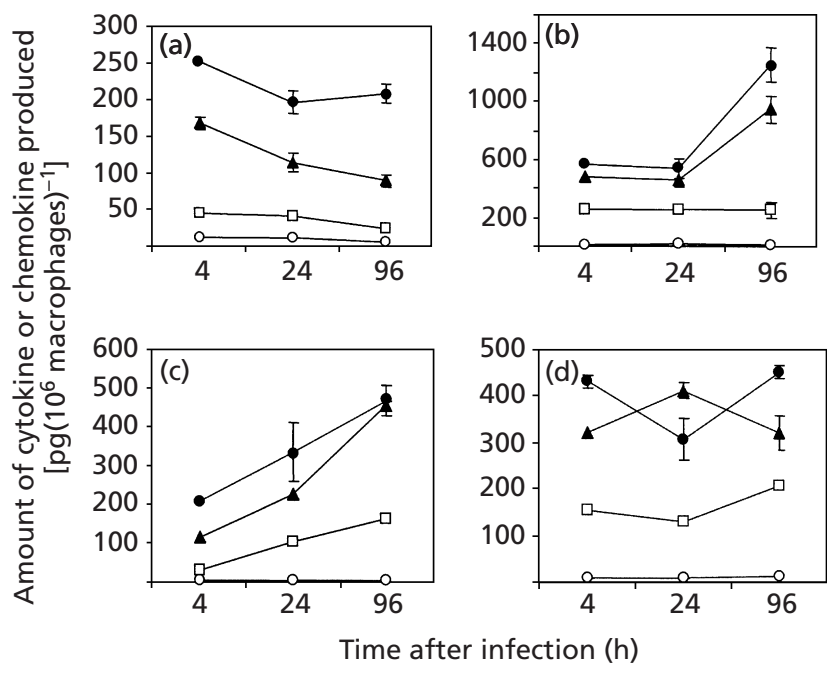

Fig. 5. Cytokine and chemokine production by BMMs infected with mycobacteria. C57BL/6 BMMs were infected with native MTB (৩), delipidated MTB ( $\square$ ), delipidated MTB reconstituted with TDM $(\boldsymbol{A})$ or they were left uninfected $(O)$. The production of (a) IL- $1 \beta$, (b) TNF- $\alpha$, (c) IL- 6 and (d) MIP- $1 \alpha$ was measured by ELISA. Data are expressed as the mean \pm one SD for three replicates.

\section{Delipidation of MTB alters the cytokine and chemokine profile of BMMs}

The production of IL- $1 \beta$, TNF- $\alpha$ and IL- 6 by the BMMs was quantified by ELISA, following their infection with mycobacteria. The level of IL- $1 \beta$ in the culture supernatants was significantly higher $(P<0 \cdot 001)$ in BMMs infected with native MTB than in BMMs infected with delipidated MTB at all time points examined (Fig. 5a). IL- $1 \beta$ production by infected BMMs was at its greatest immediately after infection $\left[251 \mathrm{pg} \quad\left(10^{6}\right.\right.$ macrophages $)^{-1}$, with only a moderate decrease observed at later time points. In contrast, IL- $1 \beta$ production by BMMs infected with delipidated MTB was not significantly different from the constitutive levels obtained in uninfected control cells. The reconstitution of delipi- dated MTB with purified TDM restored the IL- $1 \beta$ response to an intermediate level which remained significantly higher than that observed for delipidatedMTB-infected BMMs $(P<0 \cdot 001)$.

TNF- $\alpha$ protein production was also measured (Fig. $5 \mathrm{~b}$ ). The BMMs produced a high level of TNF- $\alpha$ by $4 \mathrm{~h}$ after infection with native MTB [568 pg $\left(10^{6} \text { macrophages }\right)^{-1}$ ] and this level continued to rise during the course of the infection [1255 pg $\left(10^{6} \text { macrophages }\right)^{-1}$ at $96 \mathrm{~h}$ ]. NativeMTB-infected BMMs produced significantly more TNF- $\alpha$ than BMMs infected with delipidated organisms $(P<0.001$ at all time points). Furthermore, the addition of purified TDM to delipidated MTB restored the level of TNF- $\alpha$ production by the BMMs to levels that were statistically indistinguishable from those elicited by native MTB.

The BMMs produced a high level of IL- 6 protein upon infection. The production of this protein began immediately after infection [206 pg $\left(10^{6} \text { macrophages }\right)^{-1}$ ] with native MTB and increased through the $96 \mathrm{~h} \mathrm{[468} \mathrm{pg}$ $\left(10^{6} \text { macrophages }\right)^{-1}$; Fig. $\left.5 \mathrm{c}\right]$. BMMs infected with delipidated MTB did not produce IL-6 initially; however, by $24 \mathrm{~h}$ after infection the level of IL- 6 was detectable $\left[102 \mathrm{pg}\left(10^{6} \text { macrophages }\right)^{-1}\right]$ and its levels rose through the $96 \mathrm{~h}$. In all cases, the level of protein production in the delipidated-MTB-infected macrophages remained significantly lower than in the nativeMTB-infected cells $(P<0 \cdot 01$ at all time points). The addition of purified TDM to delipidated MTB restored increases in IL-6 production that closely resembled the pattern seen for native-MTB-infected BMMs; this level was significantly elevated $(P<0 \cdot 001)$ when compared to the levels of IL-6 produced by delipidated-MTB-infected cells.

The ability of native-MTB- and delipidated-MTBinfected BMMs to produce the chemokine MIP- $1 \alpha$ was also examined. C57BL/6 BMMs infected with native MTB produced a high level of MIP- $1 \alpha$ immediately after infection; this level transiently decreased at $24 \mathrm{~h}$ before rising again at $96 \mathrm{~h}$ (Fig. 5d). Conversely, BMMs infected with delipidated MTB produced significantly less MIP$1 \alpha(P<0.001$ at $96 \mathrm{~h})$, with only a slight increase in its level by $96 \mathrm{~h}$ after infection. Although this level was reduced when compared to infection by native organisms, responses to the delipidated organisms remained statistically greater than responses to the uninfected controls. At all times examined the infection of BMMs with delipidated MTB reconstituted with purified TDM induced an intermediate level of MIP- $1 \alpha$ protein production that was significantly higher $(P<0.01)$ than that seen upon infection of the BMMs with delipidated MTB.

\section{Altered IL-12 and IL-10 production by BMMs following infection with delipidated MTB}

Delipidated-MTB-infected BMMs exhibited altered IL12 and IL-10 production compared to that produced upon infection of BMMs with native MTB. In general, the BMMs produced more IL-12 than IL-10 after 
Table 1. IL-12 and IL-10 production by BMMs infected with mycobacteria

IL-12 and IL-10 production was measured by ELISA at 4, 24 and $96 \mathrm{~h}$ after infection of C57BL/6 BMMs. Data are shown as pg protein $\left(10^{6} \text { macrophages }\right)^{-1} \pm$ one SD.

\begin{tabular}{|c|c|c|c|c|c|c|}
\hline \multirow[t]{2}{*}{ BMMs infected with } & \multicolumn{3}{|c|}{ IL-12 production $\left[\mathrm{pg}\left(10^{6} \text { macrophages }\right)^{-1}\right]$ at } & \multicolumn{3}{|c|}{ IL-10 production $\left[\mathrm{pg}\left(10^{6} \text { macrophages }\right)^{-1}\right]$ at } \\
\hline & $4 \mathrm{~h}$ & $24 \mathrm{~h}$ & $96 \mathrm{~h}$ & $4 \mathrm{~h}$ & $24 \mathrm{~h}$ & $96 \mathrm{~h}$ \\
\hline Native MTB & $582 \pm 13$ & $660 \pm 17$ & $1120 \pm 130$ & $363 \pm 52$ & $360 \pm 31$ & $561 \pm 38$ \\
\hline Delipidated MTB & $284 \pm 14$ & $421 \pm 73$ & $508 \pm 25$ & $323 \pm 30$ & $369 \pm 16$ & $408 \pm 62$ \\
\hline Delipidated MTB + TDM & $362 \pm 71$ & $745 \pm 60$ & $903 \pm 62$ & $321 \pm 50$ & $363 \pm 26$ & $520 \pm 21$ \\
\hline
\end{tabular}

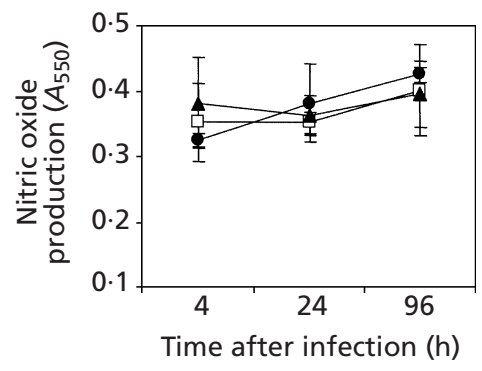

Fig. 6. Nitric oxide production by BMMs infected with mycobacteria. Nitric oxide was measured with the Griess assay at 4, 24 and $96 \mathrm{~h}$ after infection. C57BL/6 BMMs were infected with native MTB ( $)$, delipidated MTB ( $\square$ ) or delipidated MTB reconstituted with TDM $(\boldsymbol{A})$. Constitutive nitric oxide production by uninfected BMMs (mean $A_{550}=0.025$ ) was subtracted from the experimental groups; values are expressed as the mean absorbance readings at $550 \mathrm{~nm} \pm$ one SD.

infection with native MTB (Table 1). Infection with native MTB yielded IL-12 production that increased throughout the $96 \mathrm{~h}\left[1120 \mathrm{pg}\left(10^{6} \text { macrophages }\right)^{-1}\right]$. BMMs infected with delipidated organisms produced markedly less IL-12 ( $P<0.005$ at all time points $)$, although increasing amounts of this protein were evident by $96 \mathrm{~h}$. Reconstitution with TDM restored IL-12 production to levels similar to those seen after infection with native organisms. Infection of the BMMs with native organisms also produced IL-10, with its levels increasing throughout the $96 \mathrm{~h}$. The removal of surface lipids significantly reduced the amount of IL-10 produced $(P<0.05$ at $96 \mathrm{~h}$ ), but reconstitution experiments restored IL-10 responses.

\section{Removal of surface lipids does not affect the nitric oxide response to MTB}

Nitric oxide production was monitored using the Griess assay to measure nitrite in culture supernatants (Fig. 6). BMMs produced similar levels of nitric oxide when infected with the three mycobacterial groups (i.e. native, delipidated and reconstituted), with no significant differences seen between the three groups at any time after the infection of the BMMs. Nitric oxide production was evident by $4 \mathrm{~h}$ after infection, with moderate increases observed throughout the $96 \mathrm{~h}$.

\section{DISCUSSION}

The contribution of mycobacterial surface lipids and TDM to primary macrophage responses during MTB infection was investigated. BMMs were chosen for experimentation because of the relative ease in obtaining sufficient quantities of purified populations for study compared to those obtained via lung lavage. BMMs infected with native MTB produce high levels of inflammatory molecules (Jagannath et al., 2000), similar to mediators produced during TDM-induced pulmonary immunopathology in C57BL/6 mice (Perez et al., 1994, 2000). The cytokine and chemokine profiles are also remarkably similar to those elicited immediately following acute MTB infection (Actor et al., 1999, 2000; Watson et al., 2000). We have demonstrated here that BMM responses were significantly diminished, but not eliminated, when delipidated organisms were used for the infection of BMMs, suggesting that surface glycolipids are a critical component involved in macrophage responses, and the ensuing immunopathology, to MTB in vivo.

Multiple methods have been used to remove surface lipids and glycolipids from MTB. Chloroform $/$ methanol extraction is efficient but renders the organisms non-viable (Silva et al., 1985). Conversely, petroleum ether extraction allows organisms to survive following delipidation, with the growth of delipidated organisms in broth indistinguishable from that of native organisms (Silva et al., 1985). Organisms retain their acid-fast nature, presumably due to the removal of exposed glycolipids only. Silva et al. (1985) found by HPLC analysis that petroleum ether extracts of mycobacteria contain primarily TDM ( $>95 \%$ of the total extract), with only relatively small quantities of free mycolic acid glycerides, menaquinones and hydrocarbons present. TLC analysis performed in this study also demonstrated TDM as the primary extracted component. To specifically examine the influence of TDM, we replenished the mycobacterial surface with the purified molecule. Our central assumption - based on the hydrophobic nature of TDM chemistry - predicted the interaction of TDM with the mycobacterial surface in a manner consistent with natural TDM associations. Reconstitution of delipidated MTB with purified glycolipid restored macrophage responses. Although postulated, we cannot 
confirm the repopulation of the organism's surface with TDM in a confirmation mimicking that of native TDM. Indeed, other active compounds or small molecular mass lipids or proteins in the mycobacterial cell wall may have been removed or altered, since reconstitution of delipidated mycobacteria with purified TDM did not completely restore the native phenotype. This is most clearly noted in the partial recovery of the IL- $1 \beta$ response following the reconstitution experimentation.

TDM inhibits fusion between phospholipid vesicles in vitro (Spargo et al., 1991). We hypothesized that delipidated MTB would exhibit reduced viability in macrophages, possibly due to an accelerated maturation of organism-containing phagosomes to lysosomes. Initially, the growth of delipidated MTB in C57BL/6 BMMs was comparable to the growth of native MTB; however, by $96 \mathrm{~h}$ after infection the BMMs had significantly reduced the number of intracellular delipidated organisms. Removal of surface lipids can potentially affect the uptake of organisms by macrophages, thus altering the outcome of intracellular compartmentalization and survival once inside the host cell. Mycobacterial uptake by macrophages is mediated by multiple mechanisms including complement receptors, scavenger receptors and mannose receptors. However, no differences were evident in the total c.f.u. values obtained for native, delipidated or reconstituted organisms at $4 \mathrm{~h}$ after infection.

Specific innate responses by primary macrophages to TDM during in vivo MTB infection have not been thoroughly investigated. Purified TDM, emulsified in oil or coated onto hydrophobic microspheres, induces pulmonary granulomatous lesions, procoagulant activity and TNF- $\alpha$, IL- $1 \beta$ and IL- 6 mRNA production in mice (Perez et al., 1994, 2000; Behling et al., 1993). These activities have been, in part, ascribed to macrophages (Perez et al., 2000) but they have not yet been confirmed in vivo. We hypothesized that the extraction of TDM from MTB would result in reduced cytokine production by macrophages. Indeed, the removal of MTB surface lipids severely depressed inflammatory cytokine responses - an effect that was most striking in the TNF- $\alpha$ and IL- 6 responses. Reconstitution of delipidated MTB with purified TDM restored these responses. Interestingly, IL-6 is evident at $96 \mathrm{~h}$ after infection with delipidated MTB, presumably due to natural reconstitution of surface lipids by surviving organisms. Delipidation may also expose lipoarabinomannan (LAM): purified LAM modulates macrophages by down-regulating TNF- $\alpha$ (Chatterjee et al., 1992; Roach et al., 1993), IL-6 (Dahl et al., 1996) and IL-12 (Yoshida \& Koide, 1997) production. Our results are only partially consistent with increased LAM exposure. Hyporesponsiveness due to LAM may be critical for the survival of MTB in macrophages; however, increased killing of delipidated MTB was observed in C57BL/6 BMMs. Furthermore, LAM down-regulates macrophage IL-10 production (Dahl et al., 1996) - the IL-10 response was not affected by delipidation. It is therefore likely that the biological effects of the delipidation of MTB are primarily due to the loss of TDM and not to increased LAM exposure.

The role of IL- 6 in mycobacterial infection has been studied, with conflicting results. Ladel et al. (1997) showed that mice genetically deficient in IL-6 succumbed to intravenous infection with MTB more rapidly than wild-type mice. VanHeyningen and colleagues suggested that IL-6 plays an inhibitory role in antigen presentation and subsequent T-cell activation (VanHeyningen et al., 1997). MTB-infected C57BL/6 mice show a high induction of IL- 6 mRNA that coincides with granuloma development and protective immunity (Actor et al., 1999). In this study, C57BL/6 BMMs infected with native MTB produced copious amounts of IL-6. Furthermore, the level of IL- 6 induced by MTB in the BMMs was significantly higher than the levels induced by delipidated MTB, a response that was restored when these organisms were reconstituted with TDM, suggesting that TDM by itself may be sufficient for IL-6 induction in C57BL/6 macrophages.

IL-12 production is a critical innate response for the maintenance and control of tuberculosis disease and the granulomatous response (Cooper et al., 1995, 1997; Seder, 1995). Furthermore, the relative ratios of IL-12 and IL-10 are significant components influencing the outcome and immunopathology of infection (Seah et al., 2000). IL-12 protein production during infection of the BMMs with delipidated organisms was significantly reduced. Consequently, the relative ratio of IL-12:IL-10 was lowered. Reconstitution of the delipidated organisms with TDM restored the relative IL-12: IL-10 ratios. This suggests a contributory role for TDM and surface lipids in the induction of an environment leading to a Th1 response, a concept that has also been proposed by Yamagami et al. (2001) in examining TDM-specific hypersensitivity in athymic mice.

Mice demonstrate increased MIP- $1 \alpha$ production during acute MTB infection (Actor et al., 1999, 2001), presumably for the recruitment of the $\mathrm{T}$ cells and monocytes necessary for the development of mature granulomas. In this study delipidated MTB induced less MIP- $1 \alpha$ production in the BMMs than when they were infected with native MTB. Normal levels of MIP- $1 \alpha$ production were restored when delipidated organisms were reconstituted with TDM. Hence, TDM may play a role in the recruitment of $\mathrm{T}$ cells and monocytes during the early stages of infection.

In concert with the TDM-mediated inhibition of fusion of phospholipid vesicles (Spargo et al., 1991), we investigated whether TDM could also promote the intracellular survival of MTB by suppressing macrophage nitric oxide production (Garcia et al., 2000). If true, we expected delipidated MTB to generate more nitric oxide in BMMs than when they were infected with native MTB. However, this was not the case. Infection with native MTB and delipidated MTB generated nearly identical amounts of nitric oxide in the BMMs. The regulation of nitric oxide production is a complex issue. Oswald et al. (1997, 1999) demonstrated that inducible 
nitric oxide synthase mRNA in macrophages is, in part, regulated by TNF- $\alpha$ and IL-12. A reduction in priming by IL-12 should have therefore led to decreased nitric oxide production. We are further investigating nitricoxide-independent bactericidal mechanisms, specifically by examining the role of TDM in intracellular trafficking events.

In summary, the data presented here describe the responses of C57BL/6 BMMs to infection with delipidated MTB. The BMMs exhibited relatively high production of IL- $1 \beta$, TNF- $\alpha$, IL-6, IL-12 and MIP- $1 \alpha$ in response to infection with $\mathrm{MTB}$; infection with delipidated organisms abrogated these responses. By using reconstitution experiments, we determined these responses to be directly related to TDM.

\section{ACKNOWLEDGEMENTS}

This work was supported primarily by research grants from the NIH, RO1 HL68537-01 and RO1 HL68520-01. We thank Dr Chinnaswamy Jagannath for advice and helpful discussions regarding the manuscript, and Margaret Olsen for her expert technical assistance.

\section{REFERENCES}

Actor, J. K., Olsen, M., Jagannath, C. \& Hunter, R. L. (1999). Relationship of survival, organism containment, and granuloma formation in acute murine tuberculosis. J Interferon Cytokine Res 19, 1183-1193.

Actor, J. K., Leonard, C. D., Watson, V. E., Wells, A., Jagannath, C., Hunter, R. L., Jr \& Dasgupta, A. (2000). Cytokine mRNA expression and serum cortisol evaluation during murine lung inflammation induced by Mycobacterium tuberculosis. Comb Chem High Throughput Screen 3, 343-351.

Actor, J. K., Breij, E., Wetsel, R. A., Hoffmann, H., Hunter, R. L., Jr \& Jagannath, C. (2001). A role for complement C5 in organism containment and granulomatous response during murine tuberculosis. Scand J Immunol 53, 464-474.

Behling, C. A., Perez, R. L., Kidd, M. R., Staton, G. W., Jr \& Hunter, R. L. (1993). Induction of pulmonary granulomas, macrophage procoagulant activity, and tumor necrosis factor-alpha by trehalose glycolipids. Ann Clin Lab Sci 23, 256-266.

Bloch, H. (1950). Studies on the virulence of tubercle bacilli: isolation and biological properties of a constituent of virulent organisms. J Exp Med 91, 197-217.

Chatterjee, D., Roberts, A. D., Lowell, K., Brennan, P. J. \& Orme, I. M. (1992). Structural basis of capacity of lipoarabinomannan to induce secretion of tumor necrosis factor. Infect Immun 60, 1249-1253.

Cooper, A. M., Roberts, A. D., Rhoades, E. R., Callahan, J. E., Getzy, D. M. \& Orme, I. M. (1995). The role of interleukin-12 in acquired immunity to Mycobacterium tuberculosis infection. Immunology 84, 423-432.

Cooper, A. M., Magram, J., Ferrante, J. \& Orme, I. M. (1997). Interleukin 12 (IL-12) is crucial to the development of protective immunity in mice intravenously infected with Mycobacterium tuberculosis. J Exp Med 186, 39-45.

Dahl, K. E., Shiratsuchi, H., Hamilton, B. D., Ellner, J. J. \& Toossi, Z. (1996). Selective induction of transforming growth factor beta in human monocytes by lipoarabinomannan of Mycobacterium tuberculosis. Infect Immun 64, 399-405.
Falk, L. A. (1994). Isolation of bone marrow-derived macrophages. In Current Protocols in Immunology, pp. 14.0.3-14.1.9. Edited by J. E. Coligan, A. M. Kruisbeck, D. H. Margulies, E. M. Shevach \& W. Strober. New York: Wiley.

Garcia, I., Guler, R., Vesin, D., Olleros, M. L., Vassalli, P., Chvatchko, Y., Jacobs, M. \& Ryffel, B. (2000). Lethal Mycobacterium bovis Bacillus Calmette-Guerin infection in nitric oxide synthase 2-deficient mice: cell-mediated immunity requires nitric oxide synthase 2. Lab Invest 80, 1385-1397.

Hamasaki, N., Isowa, K., Kamada, K., Terano, Y., Matsumoto, T., Arakawa, T., Kobayashi, K. \& Yano, I. (2000). In vivo administration of mycobacterial cord factor (trehalose 6,6'-dimycolate) can induce lung and liver granulomas and thymic atrophy in rabbits. Infect Immun 68, 3704-3709.

Jagannath, C., Hoffman, H., Sepulveda, E., Actor, J. K., Wetsel, R. A. \& Hunter, R. L. (2000). Hypersusceptibility of A/J mice to tuberculosis is in part due to a deficiency of the fifth complement component (C5). Scand J Immunol 52, 369-379.

Jarlier, V. \& Nikaido, H. (1994). Mycobacterial cell wall : structure and role in natural resistance to antibiotics. FEMS Microbiol Lett $123,11-18$.

Kolattukudy, P. E., Fernandes, N. D., Azad, A. K., Fitzmaurice, A. M. \& Sirakova, T. D. (1997). Biochemistry and molecular genetics of cell-wall lipid biosynthesis in mycobacteria. Mol Microbiol 24, 263-270.

Ladel, C. H., Blum, C., Dreher, A., Reifenberg, K., Kopf, M. \& Kaufmann, S. H. (1997). Lethal tuberculosis in interleukin-6deficient mice. Infect Immun 65, 4843-4849.

Middlebrook, G., Dubos, R. J. \& Pierce, C. (1947). Virulence and morphological characteristics of mammalian tubercle bacilli. $J$ Exp Med 86, 175-187.

Noll, H., Bloch, H., Asselineau, J. \& Lederer, E. (1956). The chemical structure of the cord factor of Mycobacterium tuberculosis. Biochim Biophys Acta 20, 299-309.

Oswald, I. P., Dozois, C. M., Petit, J. F. \& Lemaire, G. (1997). Interleukin-12 synthesis is a required step in trehalose dimycolateinduced activation of mouse peritoneal macrophages. Infect Immun 65, 1364-1369.

Oswald, I. P., Dozois, C. M., Fournout, S., Petit, J. F. \& Lemaire, G. (1999). Tumor necrosis factor is required for the priming of peritoneal macrophages by trehalose dimycolate. Eur Cytokine Netw 10, 533-540.

Perez, R. L., Roman, J., Staton, G. W., Jr \& Hunter, R. L. (1994). Extravascular coagulation and fibrinolysis in murine lung inflammation induced by the mycobacterial cord factor trehalose-6, 6'dimycolate. Am J Respir Crit Care Med 149, 510-518.

Perez, R. L., Roman, J., Roser, S., Little, C., Olsen, M., Indrigo, J., Hunter, R. L. \& Actor, J. K. (2000). Cytokine message and protein expression during lung granuloma formation and resolution induced by the mycobacterial cord factor trehalose-6,6'-dimycolate. J Interferon Cytokine Res 20, 795-804.

Roach, T. I., Barton, C. H., Chatterjee, D. \& Blackwell, J. M. (1993). Macrophage activation: lipoarabinomannan from avirulent and virulent strains of Mycobacterium tuberculosis differentially induces the early genes c-fos, $\mathrm{KC}$, JE, and tumor necrosis factoralpha. J Immunol 150, 1886-1896.

Seah, G. T., Scott, G. M. \& Rook, G. A. (2000). Type 2 cytokine gene activation and its relationship to extent of disease in patients with tuberculosis. J Infect Dis 181, 385-389.

Seder, R. A. (1995). The role of IL-12 in the regulation of Th1 and Th2 differentiation. Res Immunol 146, 473-476.

Silva, C. L., Ekizlerian, S. M. \& Fazioli, R. A. (1985). Role of cord 
factor in the modulation of infection caused by mycobacteria. Am J Pathol 118, 238-247.

Spargo, B. J., Crowe, L. M., Ioneda, T., Beaman, B. L. \& Crowe, J. H. (1991). Cord factor ( $\alpha, \alpha$-trehalose $6,6^{\prime}$-dimycolate) inhibits fusion between phospholipid vesicles. Proc Natl Acad Sci US A 88, 737-740.

Sutterwala, F. S., Noel, G. J., Clynes, R. \& Mosser, D. M. (1997). Selective suppression of interleukin-12 induction after macrophage receptor ligation. J Exp Med 185, 1977-1985.

VanHeyningen, T. K., Collins, H. L. \& Russell, D. G. (1997). IL-6 produced by macrophages infected with Mycobacterium species suppresses T cell responses. J Immunol 158, 330-337.

Watson, V. E., Hill, L. L., Owen-Schaub, L. B., Davis, D. W., McConkey, D. J., Jagannath, C., Hunter, R. L., Jr \& Actor, J. K.
(2000). Apoptosis in Mycobacterium tuberculosis infection in mice exhibiting varied immunopathology. J Pathol 190, 211-220.

Yamagami, H., Matsumoto, T., Fujiwara, N., Arakawa, T., Kaneda, K., Yano, I. \& Kobayashi, K. (2001). Trehalose 6,6'-dimycolate (cord factor) of Mycobacterium tuberculosis induces foreignbody- and hypersensitivity-type granulomas in mice. Infect Immun 69, 810-815.

Yoshida, A. \& Koide, Y. (1997). Arabinofuranosyl-terminated and mannosylated lipoarabinomannans from Mycobacterium tuberculosis induce different levels of interleukin-12 expression in murine macrophages. Infect Immun 65, 1953-1955.

Received 31 December 2001; revised 20 March 2002; accepted 21 March 2002. 